\title{
Pulmonary tuberculosis and immunological profile of HIV/ AIDS patients in Far West Nepal
}

Bohara MS

Madan Singh Bohara

Department of Microbiology, Shree Siddhanath Science Campus (Tribhuvan University), Mahendranagar, Nepal

\section{Abstract}

Background: Tuberculosis is itself a major public health problem in Nepal and the emergence HIV further complicated the issue. Tuberculosis is the most common cause of death among HIV/AIDS patients worldwide. Tuberculosis and HIV co-infection is associated with special diagnostic and therapeutic challenges and constitutes an immense burden on healthcare system of heavily infected countries like Nepal.

Objective: The objective of the study was to determine the prevalence of pulmonary tuberculosis and their immunologic profiles among HIV positive patients.

Methods: A hospital based cross-sectional study was conducted from Januray 2012 to July 2012 among adult HIV-positive patients attending ART centers at Seti Zonal Hospital and Mahakali Zonal Hospital. Clinical and laboratory investigations including Acid Fast staining and culture were used to identify tuberculosis cases. Blood samples were collected to determine CD4+ lymphocyte count. A structured questionnaire was used to collect socio-demographic characteristics of study participants. The data was entered and analysed using SPSS version 16 software.

Results: A total of 103 HIV positive participants were enrolled. Five (4.9\%) of the study participants (three females and two males) were found to have pulmonary tuberculosis. Majority [4(80\%)] of TB-HIV co-infection was found in 21-40 years age group. Similarly four (80\%) of them were illiterate. Among five TB cases only two cases were detected by direct microscopy. In general it was observed that various clinical manifestations such as cough, chest pain, weight loss, loss of appetite etc. are more common in TB co-infected HIV patients in comparison to non-co-infected counterparts. Similarly, higher percentage of TB cases were seen in patients with low CD4+ count $\left(<500 / \mathrm{mm}^{3}\right)$.

Conclusions: We found high prevalence of tuberculosis-HIV co-infection. More such cases were seen among those with low CD4+ cell count. Early detection of co-infection is very necessary to facilitate early ART initiation, thereby strengthening their immune status.

Key words: AIDS, CD4 count, HIV, Infection, Tuberculosis

\section{INTRODUCTION}

$\mathrm{T}$ uberculosis (TB) and human immune deficiency virus (HIV) infections are two major public health problems in many parts of the world. The prevalence of TB-HIV co-infection is high worldwide and $90 \%$ of these co-infected cases live in developing nations ${ }^{1-3}$. Tuberculosis is the most common opportunistic disease and cause of death among those infected with HIV . Similarly, HIV infection is one of the most important risk factors associated with an increased risk of latent TB infection progressing to active $\mathrm{TB}^{4}$.

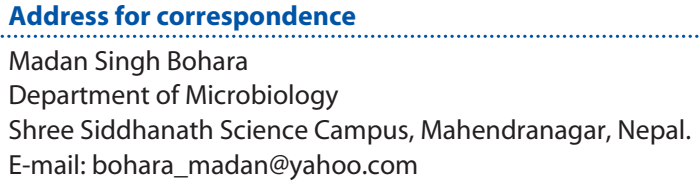

There were an estimated 1.1 million HIV positive new TB cases globally in 2011 . Around $79 \%$ of these people live in sub-Saharan Africa. In 2011 some 430,000 people died of HIV-associated TB5 ${ }^{5}$ WHO report published in2010 estimated that The South-East Asia Region accounts for nearly $15 \%$ of the global burden of new HIV-positive tuberculosis cases ${ }^{6}$.

HIV and TB have been described as the "Diabolical Duet". The reason is that the two go together. When someone is infected with HIV, the virus weakens their immune system making them more susceptible to infections. The prevalence of HIV is rapidly rising in Nepal. Due to lack of vaccines and/or effective easily accessible therapies, the disease is posing a lifelong devastation not only to the individual but also to the society. Nepal's socio-economic status, prevailing norms and values, cultural myths on 
sex and sexualities and huge population of marginalised communities make it extremely vulnerable to the HIV/ STIs epidemic. Furthermore, the high migration rate, more particularly as we see in hilly regions of Western Nepal, is still a major factor for steep increase of AIDS epidemic in Nepal?

Tuberculosis was found to be the major opportunistic infection with a prevalence of $27.3 \%$ among HIV/AIDS patients in Seti Zonal Hospital, Dhangadi ${ }^{8}$. Far-West Region of Nepal has relatively high burden of HIV/AIDS in the country ${ }^{9}$ but the specific research, especially based on the two urban areas of Far-West Region (i.e. Kailali and Kanchanpur districts) has not been done yet. It is recognised that joint TB-HIV interventions will clearly require additional data to improve both TB and HIV program performance and coverage, increase testing and counselling, prevent mother to child transmission of HIV infection, provide community home based care for people living with HIV/AIDS and provide anti-retroviral treatment. Despite these needs, there is no adequate and recent data in Far West Nepal especially in this study area. Therefore, the aim of this study was to determine the prevalence of PTB among HIV positive patients and their immunologic profiles which are important for health professionals and policy makers to make evidence based decisions.

\section{METHODS}

\section{Study Design, Period and Setting}

A hospital based cross sectional study was conducted from January 2012 to July 2012 at Department of Microbiology, Siddhanath Science Campus, Mahendranagar, Kanchanpur. Sample size calculation was done on the basis of prevalence data from a recent study $^{8}$. Altogether 103 people living with HIV/ AIDS (PLWHA) volunteers (both symptomatic and asymptomatic) were included in the study. Sampling sites were the antiretroviral therapy centres of the two government hospitals of Kailali and Kanchanpur districts - Seti Zonal Hospital, Dhangadi and Mahakali Zonal Hospital, Mahendranagar, respectively.

\section{Source Population and Study Participants}

The source population for this study were all HIV positive individuals who have the access to be served at Seti Zonal Hospital, Dhangadi and Mahakali Zonal Hospital, Mahendranagar. The study participants were all HIV positive individuals who visited ART clinic at above hospitals during the study period. Patient selection was done by random sampling method using the patients' lists available in the respective sites. Permisssion was taken from Mahakali Zonal Hospital, Mahendranagar and Seti Zonal Hospital, Dhangadi. Verbal and written consent was taken from each patient.

\section{Data Collection Procedures}

All participant sunderwent TB screening which included: (1) symptom screening (2) patients having sputum production and clinical manifestations were requested to provide three sputum specimens (spot morning sample) for smear microscopy and culture. All the specimens were transported to the Department of Microbiology, Shree Siddhanath Science Campus, Mahendranagar and specimen processing was done as per standard microbiological operating procedure. Investigation of TB was done by acid fast bacilli (AFB) culture by modified Petroff's method using Loweinstein Jensen medium, and direct microscopy of AFB stained smear by Ziehl-Neelsen method as per WHO protocol ${ }^{10}$. To eliminate wrong evaluation, quality control of the sputum was done for possible cases. If another sampling was possible, specimen without mucopurulent part was rejected and asked for another sample. Study participants were interviewed about the presence of clinical manifestations of TB. Socio-demographic characteristics and other related risk factors were collected using structured questionnaire. Five $\mathrm{ml}$ of venous blood was aseptically collected in ethylene-diamine-tetra-acetic acid (EDTA) tubes for CD4+ lymphocyte count. The CD4+ lymphocyte count was done by BD FACS count flow cytometry machine ${ }^{11}$. The daily, weekly and monthly maintenance of the BD FACS count flow cytometry was done according to the instruments manual and quality control for both reagent and the machine was done daily. Study participants were interviewed about the presence of clinical manifestations of TB. Sociodemographic characteristics and other related risk factors were collected using structured questionnaire.

\section{Case Definitions}

Smear positive PTB was defined as one or more sputum smear examinations positive for acid fast bacilli (AFB). Smear-negative PTB was defined as three sputum smear examinations negative for AFB but with evidence of TB from chest $x$-ray or other investigations. The rough, tough and buff coloured colonies of Mycobacterium tuberculosis developed on L.J. medium up to eight weeks was defined as culture positive $^{10}$. 


\section{Data Analysis}

Data were checked for completeness and entered and analysed using SPSS version 16 (SPSS Inc. Chicago, USA). Data were summarised using frequency tables.

\section{RESULTS}

This study revealed the prevalence of TB in HIV/AIDS patients in Far West Nepal. Among 103 confirmed seropositive HIV/AIDS patients examined, five (4.9\%) were positive for Acid Fast Bacili (AFB) showing a prevalence rate of $4.9 \%$ pulmonary tuberculosis in HIV/AIDS patients. Out of 103 study participants, 46 (44.7\%) were males and 57 (55.3\%) were females.

The lowest and the highest numbers of the study participants were five (4.9\%) in age group 1-10 years and 37 (35.9\%) in age group 31-40 years respectively. Majority of participants (40.8\%) were illiterate while only one had studied upto bachelor's level [Table 1].

\section{TB-HIV Co-Infection}

Five $(4.9 \%)$ of the study participants were found to have pulmonary tuberculosis out of which three were female and two were male.

The majority of TB-HIV co-infection found in age groups 21-30 years and age group 31-40 years (two in each). TB-HIV co-infection was more common [four (80\%)] in illiterate patients while remaining one had primary level education. Smoking and alcohol consumption did not show statistically significant association with TB-HIV coinfection [Table 1].

Table 1: Baseline characteristics of HIV/AIDS Patients in Far West Nepal

\begin{tabular}{|c|c|c|c|c|c|}
\hline \multirow{2}{*}{ Characteristics } & & \multicolumn{3}{|c|}{ Pulmonary TB } & \multirow[b]{2}{*}{ p-value } \\
\hline & & $\begin{array}{l}\text { Present (TB-HIV co-infection) } \\
\qquad n=5\end{array}$ & $\begin{array}{l}\text { Absent (only HIV) } \\
n=98\end{array}$ & $\begin{array}{l}\text { Total tested } \\
\qquad=103\end{array}$ & \\
\hline \multirow{6}{*}{ Age (years) } & $1-10$ & 0 & $5(5.1 \%)$ & $5(4.9 \%)$ & \\
\hline & $11-20$ & 0 & $6(6.1 \%)$ & $6(5.8 \%)$ & \\
\hline & $21-30$ & $2(40.0 \%)$ & 27 (27.6\%) & $29(28.2 \%)$ & \\
\hline & $31-40$ & 2 (40.0\%) & 35 (35.7\%) & 37 (35.9\%) & \\
\hline & $41-50$ & $1(20.0 \%)$ & 19 (19.4\%) & 20 (19.4\%) & \\
\hline & $51-60$ & 0 & $6(6.1 \%)$ & $6(5.8 \%)$ & \\
\hline \multirow{2}{*}{ Sex } & Male & $2(40.0 \%)$ & $44(44.9 \%)$ & 46 (44.7\%) & \\
\hline & Female & $3(60.0 \%)$ & $54(55.1 \%)$ & 57 (55.3\%) & \\
\hline \multirow{6}{*}{ Educational Status } & Illiterate & $4(80.0 \%)$ & $38(38.8 \%)$ & $42(40.8)$ & \\
\hline & Primary & $1(20.0 \%)$ & 39 (39.8\%) & 40 (38\%) & \\
\hline & Lower Secondary & 0 & $7(7.1 \%)$ & $7(6.8 \%)$ & \\
\hline & Secondary & 0 & $10(10.2 \%)$ & $10(9.7 \%)$ & \\
\hline & Higher Secondary & 0 & $3(3.1 \%)$ & $3(2.9 \%)$ & \\
\hline & Bachelor and above & 0 & $1(1.0 \%)$ & $1(1.0 \%)$ & \\
\hline \multirow{7}{*}{ Occupation } & Farming & $2(40.0 \%)$ & $31(31.6 \%)$ & $33(32.0 \%)$ & \\
\hline & Unemployed & $2(40.0 \%)$ & $21(21.4 \%)$ & $23(22.3 \%)$ & \\
\hline & I/NGO & 0 & $13(13.3 \%)$ & $13(12.6 \%)$ & \\
\hline & Student & $1(20.0 \%)$ & $10(10.2 \%)$ & $11(10.7 \%)$ & \\
\hline & Job & 0 & $10(10.2 \%)$ & $10(9.7 \%)$ & \\
\hline & Business & 0 & $9(9.2 \%)$ & $9(8.7 \%)$ & \\
\hline & Volunteer & 0 & $4(4.1 \%)$ & $4(3.9 \%)$ & \\
\hline \multirow{2}{*}{ Smoking Habit } & Yes & $1(20.0 \%)$ & $19(19.3 \%)$ & 20 (19.4\%) & \multirow{2}{*}{0.973} \\
\hline & No & 4 (80.0\%) & 79 (80.7\%) & 83 (80.6\%) & \\
\hline \multirow{2}{*}{ Alcoholic Habit } & Yes & $1(20.0 \%)$ & 9 (9.1\%) & $10(9.7 \%)$ & \multirow{2}{*}{0.493} \\
\hline & No & $4(80.0 \%)$ & 89 (90.9\%) & 93 (90.0\%) & \\
\hline \multirow{2}{*}{ Family Type } & Joint & $3(60.0 \%)$ & $63(64.2 \%)$ & 66 (64.0\%) & \\
\hline & Nuclear & 2 (40.0\%) & 35 (35.8\%) & 37 (36.0\%) & \\
\hline
\end{tabular}


Comparison between microscopically and culture was done and it was found that microscopy could detect only two of the five TB cases detected by culture (Table 2).

\section{Immunological Profile of HIV Infected Patients}

The majority of study participants [46 (44.7\%)] had CD4+ lymphocyte count of $1-200$ cells $/ \mathrm{mm}^{3}$ and 26 (28.3\%) of study participants had CD4+ lymphocyte count of 201-500 cells $/ \mathrm{mm}^{3}$. Twenty (21.7\%) of were found to have CD4+ lymphocyte count within 501-1500 cells/ $\mathrm{mm}^{3}$. The TB-HIV co-infection was highest [four (80\%)] in patients who had CD4+ cell count within 1-200 cells/ $\mathrm{mm}^{3}$ and one $(20.0 \%)$ was found to have CD4+ cell count of 501-1500 cells $/ \mathrm{mm}^{3}$. Majority of participants [61 (59.2\%)] were in WHO clinical stage II followed by WHO clinical stage I [26 (25.2\%)]. Regarding clinical sign and symptoms, cough, chest pain and loss of appetite were found to be the major sign and symptoms of TB/HIV coinfected persons followed by abdominal pain, headache and night sweat. Out of the $103 \mathrm{HIV}$ infected patients, 80 (77.7\%) were under ART. All five TB-HIV co-infected cases were under ART (table 3).

Table 2: Comparison between Microscopy and Culture Techniques for Diagnosis of Pulmonary TB in HIV/AIDS Patients

\begin{tabular}{ccc|}
\hline Pulmonary TB & Microscopy & Culture \\
Positive test & $2(2.0 \%)$ & $5(4.9 \%)$ \\
\hline Negative test & $101(98.0 \%)$ & $98(95.1 \%)$ \\
\hline Total & $\mathbf{1 0 3}$ & $\mathbf{1 0 3}$ \\
\hline
\end{tabular}

\section{DISCUSSION}

The ever-increasing prevalence of PTB in Far West Nepal has been made worse by increasing incidence of HIV/AIDS. In this study, we noted high prevalence of PTB (4.9\%) among HIV positive ART receiving patients. In a similar study conducted in Pokhara, Nepal,5.97\% patients had TB-HIV co-infection?. The finding of TBHIV co-infection of this study was in line with studies conducted in Nigeria (7.8\%), Tanzania (8.5\%) and Ethiopia $(7.5 \%)^{11-13}$. However, the prevalence in this study was lower compared to that in studies conducted in Kathmandu, Nepal (23\%) and Palpa (10.8\%) ${ }^{14,15}$. More recent studies carried out in admitted HIV/AIDS patients in Seti Zonal Hospital, Dhangadi documented 27.3\% TB prevalence ${ }^{7}$. The presence of this difference could be explained by the fact that this study considers only PTB cases but not other forms of TB. This study was also restricted to HIV positive patients attending ART centres of Seti and Mahakali Zonal hospital and other factor may be that TB-HIV co-infectionwas found only under ART patients in which relatively strong immunity might have contributed for lower prevalence of TB-HIV co-infection since opportunistic infections are more prevalent during compromised immune status. Although HIV prevalence is high in this region but HIV positive patients may ignore or not have approach to visit the ART centres. Even though smoking habit of patients are important contribution factor of TB but in this study TB-HIV coinfection was high in non-smoker and non-alcoholic patients respectively which was statistically not significant. It was supported that HIV infection increases the vulnerability of TB infection.

Table 3: Clinical and Immunological profile of HIV/AIDS patients in Far West Nepal

\begin{tabular}{|c|c|c|c|c|c|}
\hline \multirow{2}{*}{\multicolumn{2}{|c|}{ Variable }} & \multicolumn{4}{|c|}{ Pulmonary tuberculosis } \\
\hline & & $\begin{array}{c}\text { Present (TB-HIV) } \\
n=5\end{array}$ & $\begin{array}{l}\text { Absent (only HIV) } \\
\qquad n=98\end{array}$ & $\begin{array}{l}\text { Total tested } \\
\qquad N=103\end{array}$ & P-value \\
\hline \multirow{4}{*}{ WHO clinical stage } & Stage I & $1(20.0 \%)$ & $25(25.5 \%)$ & $26(25.2 \%)$ & \\
\hline & Stage II & $4(80.0 \%)$ & $57(58.2 \%)$ & $61(59.2 \%)$ & \\
\hline & Stage III & 0 & $14(14.3 \%)$ & $14(13.6 \%)$ & \\
\hline & Stage IV & 0 & $2(2.0 \%)$ & $2(1.9 \%)$ & \\
\hline \multirow{3}{*}{ CD4 count (per $\mathrm{mm}^{3}$ ) } & $1-200$ & $4(80.0 \%)$ & $42(48.3 \%)$ & $46(50.0 \%)$ & \\
\hline & $201-500$ & 0 & $26(29.9 \%)$ & $26(28.3 \%)$ & 0.288 \\
\hline & $501-1500$ & $1(20.0 \%)$ & $19(21.8 \%)$ & $20(21.7 \%)$ & \\
\hline \multirow{5}{*}{ Clinical feature } & Cough & $3(60.0 \%)$ & $29(29.6 \%)$ & $32(31.1 \%)$ & \\
\hline & Chest pain & $3(60.0 \%)$ & $22(22.4 \%)$ & $25(24.3 \%)$ & \\
\hline & Loss of appetite & $3(60 . \% 0)$ & $17(17.3 \%)$ & $20(19.4 \%)$ & \\
\hline & Weight loss & $2(40.0 \%)$ & 16 (16.3\%) & 18 (17.5\%) & \\
\hline & Night sweat & $2(40.0 \%)$ & $12(12.2 \%)$ & 14 (13.6\%) & \\
\hline \multirow{2}{*}{ ART status } & Yes & $5(100 \%)$ & $75(76.5 \%)$ & $80(77.7 \%)$ & \\
\hline & No & 0 & $23(23.5 \%)$ & $23(22.3 \%)$ & \\
\hline
\end{tabular}


One of the most important limitations of this study is that this study could not reflect the scenario of TB/HIV burden in the community level because the sampling sites are antiretroviral therapy centres of Seti Zonal Hospital and Mahakali Zonal Hospitals of Far Western Nepal. In fact, more extensive community based surveillance is recommended to get the more representative data of the TB/ HIV co-infection to develop national policy regarding this burning issue.

The socio-demographic characteristics of the patients demonstrated that relatively high proportion of females (55.3\%) attended ART sites in this region in comparison to national situation. A similar study carried out during 2009 in ten ART sites across Nepal showed lower proportion of female $(43 \%)$ than male ${ }^{14}$. TB-HIV co-infection was also high in illiterate patients, females, farmersand unemployed patients. This scenario represents the general trend of the major socioeconomic problem that lead to spread of HIV epidemic in Far Western region of Nepal suggesting that the HIV epidemic in this region is in the verge of attaining generalized form, affecting the silent population such as women (housewives) and children, and the migrant labourers acting as carrier of the virus. A previous socio-demographic study of PLWHA visiting Seti Zonal hospital ART centre suggested that most of the people of this region go to India to earn money as migrant labourers. There they acquire HIV infection and transmit the infection to their innocent housewives during their short visit to home ${ }^{6}$. The findings of our study also support this fact as the majority of the TB-HIV co-infected patients were in the productive age group 31-40 years, illiterate and lack of skills (unemployment). All these factors in association with unsafe sexual practice were found to be the major cause of HIV transmission. The age wise distribution of studied population is consistent with those of other socio-demographic studies conducted in different parts of the nation ${ }^{7,14}$.

In general it was observed that various clinical manifestations such as cough, chest pain, weight loss, loss of appetite etc. are more common in TB coinfected HIV patients in comparison to non-co-infected counterparts. Similarly, higher percentage of TB cases were seen in patients with low CD4 count $(<200)$ and
WHO clinical stage II. As CD4+ lymphocyte count decreased the body defence mechanism will be overwhelmed by various opportunistic infections. These clinical and laboratory markers could be useful for surveillance of TB/HIV co-infection. Microbiologically, the most important findings of this study is the exploration of the fact that TB case detection by culture was found to be the most effective (which detected all five cases of TB) than direct observation of AFB stained smear (which detected only two cases of TB) i.e. there is predominance of smear negative TB cases among people infected with HIV. Therefore, on the basis of this study, it can be recommended that the TB control organizations should take concrete steps regarding the inclusion of cultural techniques along with direct microscopy, at least for HIV infected persons. Furthermore, socioeconomic profile of the patients suggested that youths of productive age groups with low socioeconomic status were more vulnerable to HIV/AIDS. So, special program targeted on these marginalized communities should be launched to create public awareness through behaviour change communication/information approaches.

\section{CONCLUSION}

High prevalence of PTB was found in HIV positive patients. Co-infection was more common in those with low CD4 count though there was no statistically significant association. Early detection of co-infection is very necessary to prolong their ART initiation time and by then strengthening their immune status. Sixty percent of TB infections were smear negative. Thus culture methods should also be given priority besides microscopy for detection of TB cases.

\section{ACKNOWLEDGEMENT}

The author would like to thank University Grants Commission Nepal for providing fund to complete the work. Author is also equally thankful to Siddhanath Science Campus for permitting to use the Microbiology Lab. Special thanks and appreciation also goes to all the study participants who voluntarily participated in this study and technical staff of Seti Zonal Hospital. The author also would like to thank Mr. Govind Prasad Dhungana for helping in research work and laboratory work. 


\section{REFERENCES}

1. WHO, UCSF. Report on HIV/AIDS in Ethiopia. CDC MMWR 2003;52:217.

2. Harrington M. From HIV to tuberculosis and back again: A tale of activism in 2 pandemics. Clin Infect Dis. 2010;50:S260-S266.

3. Friedland G, Churchyard GJ, Nardell E. Tuberculosis and HIV co- infection: current state of knowledge and research priorities. J Infect Dis. 2007;196:S1-S3.

4. Meya DB, McAdam KP. The TB pandemic: an old problem seeking new solutions. J Intern Med. 2007;261:309-29.

5. World Health Organization. TB/HIV factsheet [Internet].Geneva: WHO; 2013 [cited 2013 Sep 16]. Available from:http://www.who.int/tb/challenges/ hiv/tbhiv_factsheet_2013.pdf

6. World Health Organization. HIV/AIDS in the South East Asia region: Progress report 2010 [Internet]. Geneva: WHO; 2010 [cited 2013 Sep 16]. Available from: http://aidsdatahub.org/dmdocuments/ WH_2010_HIV_AIDS_in_the_South_East_Asia_ Region_Progress_Report_2010.pdf

7. Paudel BN, Sharma S, Singh GB, Dhungana GP, Paudel P. Socio-demographic profile of HIV patients at SetiZonal Hospital. J Nep Health Res Counc. 2008;6(13):107-10.

8. Poudel BN, Dhungana GP. Scenario of HIV/AIDS patients in a government hospital of Nepal. J Nep Health Res Counc. 2010;8(2):103-6.
9. Verma SC, Dhungana GP, Joshi HS, Kunwar HB, Pokhrel AK. Prevalence of pulmonary tuberculosis among HIV infected persons in Pokhara, Nepal. J Nep Health Res Counc. 2012;10(1):32-6.

10. World Health Organization. Laboratory service in tuberculosis control, tuberculosis culture. Geneva:WHO; 1998.

11. BD FACS Count System User's Guide for Use with BD FACS Count CD4 Reagents [Internet]. 2008. Available from:http://www.bdbiosciences.com/external_files/ $\mathrm{pm} / \mathrm{doc} / \mathrm{manuals} /$ live/web enabled/339011

12. International Union against Tuberculosis and Lung Disease. Sputum examination for tuberculosis by direct microscopy in low income countries. 5th ed. Paris: IUATLD; 2000.

13. Wondimencch Y. Mulue D, Belyhun Y. Pulmonary tuberculosis and immunological profile of HIV coinfected patients in Northwest Ethiopia. BMC Res Notes. 2012;5:331.

14. Dhungana GP, Ghimere P, Sharma S, Rijal BP. Tuberculosis co-infection in HIV infected patients of Kathmandu. Nep Med Coll J. 2008;10(2):96-9.

15. Ghimire P, Dhungana JR, Bam DS, Rijal BP. Tuberculosis and Human immunodeficiency virus Co-infection in United Mission Hospital-Tansen. SAARC J Tuberculosis Lung Dis. 2004;1:32-8. 